\title{
Association of the HLA-B alleles with carbamazepine-induced Stevens-Johnson syndrome/toxic epidermal necrolysis in the Javanese and Sundanese population of Indonesia: the important role of the HLA-B75 serotype
}

\author{
Rika Yuliwulandari ${ }^{*}$ 1,2,3, Erna Kristin ${ }^{3,4}$, Kinasih Prayuni ${ }^{2}$, Qomariyah Sachrowardi ${ }^{5}$, \\ Franciscus D Suyatna ${ }^{3,6}$, Sri Linuwih Menaldi ${ }^{7}$, Nuanjun Wichukchinda ${ }^{8}$, Surakameth \\ Mahasirimongkol ${ }^{8}$ \& Larisa $\mathrm{H}_{\text {Cavallari }}{ }^{9}$ \\ ${ }^{1}$ Department of Pharmacology, Faculty of Medicine, YARSI University, Cempaka Putih, Jakarta Pusat, DKI Jakarta, Indonesia \\ ${ }^{2}$ Genomic Medicine Research Centre, YARSI Research Institute, YARSI University, Cempaka Putih, Jakarta Pusat, DKI Jakarta, \\ Indonesia \\ ${ }^{3}$ The Indonesian Pharmacogenomics Working Group, DKI Jakarta, Indonesia \\ ${ }^{4}$ Department of Pharmacology \& Therapy, Faculty of Medicine, Gadjah Mada University, Yogyakarta, Indonesia \\ ${ }^{5}$ Department of Physiology, Faculty of Medicine, YARSI University, Cempaka Putih, Jakarta Pusat, DKI Jakarta, Indonesia \\ ${ }^{6}$ Department of Pharmacology \& Therapeutic, Faculty of Medicine, University of Indonesia, Salemba, Jakarta Pusat, DKI Jakarta, \\ Indonesia \\ ${ }^{7}$ Department of Dermatology Clinic, Faculty of Medicine, University of Indonesia, Salemba, Jakarta Pusat, DKI Jakarta, Indonesia \\ ${ }^{8}$ Medical Genetics Center, Medical Life Sciences Institute, Department of Medical Sciences, Ministry of Public Health, Nonthaburi \\ 11000 , Thailand \\ ${ }^{9}$ College of Pharmacy, University of Florida, Gainesville, FL 32603, USA \\ * Author for correspondence: Tel.: +62 21 4206675; Fax: +62 21 4243171; rika.yuliwulandari@yarsi.ac.id
}

Carbamazepine (CBZ) is a common cause of life-threatening cutaneous adverse drug reactions such as Stevens-Johnson syndrome (SJS) and toxic epidermal necrolysis (TEN). Previous studies have reported a strong association between the HLA genotype and CBZ-induced SJS/TEN. We investigated the association between the HLA genotype and CBZ-induced SJS/TEN in Javanese and Sundanese patients in Indonesia. Nine unrelated patients with CBZ-induced SJS/TEN and 236 healthy Javanese and Sundanese controls were genotyped for HLA-B and their allele frequencies were compared. The HLA-B*15:02 allele was found in $66.7 \%$ of the patients with CBZ-induced SJS/TEN, but only in $29.4 \%$ of tolerant control $(p=0.029$; odds ratio [OR]: $6.5 ; 95 \% \mathrm{Cl}: 1.2-33.57)$ and $22.9 \%$ of healthy controls ( $p=0.0021 ;$ OR: $6.78 ; 95 \% \mathrm{Cl}: 1.96-$ 23.38). These findings support the involvement of $H L A-B * 15: 02$ in CBZ-induced SJS/TEN reported in other Asian populations. Interestingly, we also observed the presence of the $H L A-B * 15: 21$ allele. $H L A-B * 15: 02$ and $H L A-B^{*} 15: 21$ are members of the HLA-B75 serotype, for which a greater frequency was observed in CBZ-induced SJS/TEN (vs tolerant control [ $p=0.0078 ;$ OR: $12 ; 95 \% \mathrm{Cl}: 1.90-75.72$ ] and vs normal control [p = 0.0018; OR: 8.56; $95 \% \mathrm{Cl}: 1.83-40])$. Our findings suggest that screening for the HLA-B75 serotype can predict the risk of CBZ-induced SJS/TEN more accurately than screening for a specific allele.

First draft submitted: 5 June 2017; Accepted for publication: 26 September 2017; Published online: 20 October 2017

Keywords: carbamazepine • HLA-B75 • Indonesia • Javanese • Stevens-Johnson syndrome • Sundanese • toxic epidermal necrolysis

Carbamazepine (CBZ) is used to treat seizures, neuropathic pain such as trigeminal neuralgia, chronic pain and bipolar disorder [1,2]. CBZ is effective in most patients and is usually recommended as the first line antiepileptic. Additionally, it is available as a generic medication and thus is generally affordable [3]. Previous studies have shown that CBZ is a common cause of Stevens-Johnson syndrome (SJS) and toxic epidermal necrolysis (TEN) [4-6]. 
SJS/TEN is characterized by erythematous skin, extensive detachment of the epidermis and erosion of the mucous membranes; it is a single-disease entity which is present at different severities but with a common pathogenesis [7]. SJS/TEN patients are classified based on the extent of skin detachment: limited to less than $10 \%$ of the body surface area (BSA) in SJS, 10-30\% BSA in SJS/TEN overlap, and more than 30\% of the BSA in TEN $[1,7]$.

Multiple studies have shown the association between HLA genotype and CBZ-induced SJS/TEN [8]; some HLA alleles have been reported to be strong predictive markers for SJS/TEN [9]. The most prominent marker reported for CBZ-induced SJS/TEN is $H L A-B^{*} 15: 02$, which is commonly found in southeast Asian populations [2,5,10-16], while other studied populations were found to lack a correlation between the $H L A-B^{*}$ 15:02 genotype and SJS/TEN risk [17-19]. This indicates that the $H L A-B^{*} 15: 02$ allele is not a universal marker for CBZ-induced SJS/TEN risk, but instead may vary by ethnicity $[17,20]$. A recent study reported the case of a Thai patient who developed CBZ-induced SJS while screening negative for $H L A-B^{*} 15: 02$, but was later found to have $H L A-B^{*} 15: 21$ [21]; other HLA-B75 serotype markers similar to $H L A-B^{*} 15: 02$ are $H L A-B^{*} 15: 08,-B^{*} 15: 11$ and $-B^{*} 15: 31$ [22].

A previous study of HLA in the Javanese and Sundanese populations of Indonesia showed an $H L A-B^{*} 15: 02$ allele frequency of $11.6 \%[23]$. Because of the relatively high frequency of the $H L A-B^{*} 15: 02$ allele in these populations, it is important to determine the association between $H L A-B^{*}$ 15:02 and CBZ-induced SJS/TEN among the Javanese and Sundanese ethnic groups. Therefore, in this study, we investigated the association between the HLA-B*15:02 genotype and the B-75(15) serotype and CBZ-induced SJS/TEN in Javanese and Sundanese populations. To the best of our knowledge, this report constitutes the first study of the genetic associations of drug-induced SJS/TEN in these populations.

\section{Materials \& methods}

Genetic samples from 12 patients of Javanese and Sundanese ethnicity with CBZ-induced SJS/TEN were collected during 2014-2015 from several hospitals on Java Island. Patients were diagnosed according to Roujeau's diagnostics criteria and confirmed by dermatologist based on the clinical morphology of their skin damage [24]. The CBZtolerant control group of Javanese or Sundanese ethnicity consisted of 17 patients with epilepsy who did not show any cutaneous reaction 3 months after taking CBZ. Samples from 236 unrelated healthy individuals of Javanese or Sundanese ethnicity in the general population were obtained as part of a previous study [25] and used as normal controls. All subjects were interviewed for ethnic background for three generations back. The genomic DNA for both groups was isolated in the same manner and stored at $-20^{\circ} \mathrm{C}$ until further processing. The study protocols were approved by the Research Ethics Committees of YARSI University, Jakarta, Indonesia and YARSI collaborative hospitals, and all subjects signed informed consent forms.

HLA-B genotypes were determined using a Luminex multianalyte profiling system (xMAP; Luminex, TX, USA) with a WAKFlow HLA typing kit (Wakunaga, Hiroshima, Japan), which is based on polymerase chain reactionreverse sequence-specific oligonucleotide probes, according to the manufacturer's instructions. The HLA alleles were assigned by analyzing hybridization patterns of the target using WAKFlow HLA typing software (Wakunaga).

HLA-B genotype and HLA-B75 serotype frequencies were compared between patients and controls using $\mathrm{X}^{2}$ test. The strength of association was estimated by calculating the odds ratio (OR) and $95 \% \mathrm{CI}$. All reported p-values were two-sided, and a value of $\mathrm{p}<0.05$ was considered statistically significant. The HLA-B75 serotype was defined using the IMGT/HLA database nomenclature [22].

\section{Results}

12 patients with CBZ-induced SJS/TEN and 17 tolerant controls were recruited in this study. General population control from previous study was also included in analysis to search for an association between HLA allele and CBZ-induced SJS/TEN. All alleles with frequencies $<1 \%$ in the general population control groups were classified as $\mathrm{B}$ others (Table 1). Most of the subjects were female (53\%) and the median age was 34.25 years old (15-59 years old). The presence of $H L A-B^{*} 15: 02$ in CBZ-induced SJS/TEN (8/12; 66.7\%) was significantly more than that of the CBZ-tolerant control group (4/17; 29.4\%; $\mathrm{p}=0.029$; OR: $6.5 ; 95 \% \mathrm{CI}: 1.2-33.57)$ and than that of normal controls $(54 / 236 ; 22.9 \%$; $\mathrm{p}=0.0021$; OR: 6.78; 95\% CI: 1.96-23.38), see Table 1 . The HLA-B*18:01 allele tended to occur more often in patients, but statistical significance was not reached (33.3\% [3/9] in SJS/TEN patients compared with $5.9 \%$ in tolerant control and $12.3 \%$ in general population controls). HLA-B*44:03 and $H L A-B^{*} 15: 21$ allele frequencies were also higher in patients, but not statistically significant (Table 1). Table 2 shows the association between the HLA-B75 serotype and CBZ-induced SJS/TEN. The presence of HLA-B75 serotype was higher in CBZ-induced SJS/TEN patients (10/12; 83.3\%) than in tolerant control $(5 / 17 ; 29.4 \%)$ 
Table 1. Frequencies of heterogenous alleles of HLA-B loci.

\begin{tabular}{|c|c|c|c|c|c|c|c|}
\hline \multirow{2}{*}{ HLA-B allele } & \multicolumn{3}{|c|}{ Frequency (\%) } & \multicolumn{2}{|c|}{ Case with SJS/TEN vs general control } & \multicolumn{2}{|c|}{ Case with SJS/TEN vs tolerant control } \\
\hline & SJS $/$ TEN $(n=12)$ & $\begin{array}{l}\text { General control } \\
(n=236)^{\dagger}\end{array}$ & $\begin{array}{l}\text { Tolerant control } \\
(n=17)\end{array}$ & $\begin{array}{l}\text { Odds ratio }(95 \% \\
\mathrm{Cl})\end{array}$ & p-value & $\begin{array}{l}\text { Odds ratio }(95 \% \\
\mathrm{Cl})\end{array}$ & p-value \\
\hline 07:02 & - & $4(1.7 \%)$ & - & - & - & - & - \\
\hline 13:01 & - & $6(2.5 \%)$ & - & - & - & - & - \\
\hline 13:02 & - & $5(2.1 \%)$ & - & - & - & - & - \\
\hline 15:13 & - & $51(21.6 \%)$ & $2(11.8 \%)$ & - & - & - & - \\
\hline 15:17 & - & $4(1.7 \%)$ & - & - & - & - & - \\
\hline $15: 21$ & $2(16.7 \%)$ & $33(14 \%)$ & $1(5.9 \%)$ & $1.24(0.26-5.89)$ & 1 & $3.2(0.26-40.05)$ & 0.55 \\
\hline $15: 214$ & $1(8.3 \%)$ & - & $1(5.9 \%)$ & - & - & - & - \\
\hline $15: 25$ & - & $8(3.4 \%)$ & - & - & - & - & - \\
\hline 18:33 & - & - & $1(5.9 \%)$ & - & - & - & - \\
\hline $27: 06$ & - & $14(5.9 \%)$ & - & - & - & - & - \\
\hline 35:01 & - & - & $2(11.8 \%)$ & - & - & - & - \\
\hline $35: 02$ & - & - & $1(5.9 \%)$ & - & - & - & - \\
\hline $35: 03$ & - & $11(4.7 \%)$ & - & - & - & - & - \\
\hline 35:05 & $1(8.3 \%)$ & $39(16.5 \%)$ & $4(23.5 \%)$ & $0.42(0.053-3.36)$ & 0.49 & $0.29(0.02-3.04)$ & 0.37 \\
\hline $35: 32$ & $1(8.3 \%)$ & - & - & - & - & - & - \\
\hline $38: 02$ & - & $25(10.6 \%)$ & - & - & - & - & - \\
\hline 40:01 & - & $17(7.2 \%)$ & $1(5.9 \%)$ & - & - & - & - \\
\hline 40:06 & - & $4(1.7 \%)$ & - & - & - & - & - \\
\hline 57:01 & - & $6(2.5 \%)$ & - & - & - & - & - \\
\hline $58: 01$ & - & $27(11.4 \%)$ & - & - & - & - & - \\
\hline B others & - & $15(6.4 \%)$ & - & - & - & - & - \\
\hline $\begin{array}{l}\text { †From Javanes } \\
\text { '-' no data ava } \\
\text { SJS/TEN: Steve }\end{array}$ & danese General Pop & $\begin{array}{l}\text { ation [25]. } \\
\text { dermal necrolysis. }\end{array}$ & & & & & \\
\hline
\end{tabular}

Table 2. HLA-B75 serotype carrier frequency in carbamazepine-induced Stevens-Johnson syndrome/toxic epidermal necrolysis patients and normal controls.

\begin{tabular}{|c|c|c|c|c|c|c|c|}
\hline \multirow[t]{2}{*}{ HLA-B serotype } & \multicolumn{3}{|c|}{ Frequency (\%) } & \multicolumn{2}{|c|}{ Case with SJS/TEN vs general control } & \multicolumn{2}{|c|}{ Case with SJS/TEN vs tolerant control } \\
\hline & SJS $/$ TEN $(n=12)$ & $\begin{array}{l}\text { General control } \\
(n=236)\end{array}$ & $\begin{array}{l}\text { Tolerant control } \\
(n=17)\end{array}$ & $\begin{array}{l}\text { Odds ratio }(95 \% \\
\text { Cl) }\end{array}$ & p-value & $\begin{array}{l}\text { Odds ratio }(95 \% \\
\text { Cl) }\end{array}$ & p-value \\
\hline B75 & $10(83.3 \%)$ & $87(36.9 \%)$ & $5(29.4 \%)$ & $8.56(1.83-40)$ & 0.0018 & $12(1.90-75.72)$ & 0.0078 \\
\hline
\end{tabular}

SJS/TEN: Stevens-Johnson syndrome/toxic epidermal necrolysis. 
and general population control $(87 / 236 ; 36.9 \%)$. It was revealed that HLA-B75 allele is significantly associated with CBZ-induced SJS/TEN compared with tolerant control ( $\mathrm{p}=0.0078$; OR: 12; 95\% CI: 1.90-75.72) and general population control ( $\mathrm{p}=0.0018$; OR: 8.56; 95\% CI: $1.83-40)$.

\section{Discussion}

We determined the HLA-B genotypes in $12 \mathrm{CBZ}$-induced SJS/TEN patients, 17 CBZ-tolerant patients and 236 normal controls using a high-resolution DNA typing method. We found that $H L A-B^{*} 15: 02$ was the most frequent allele, and observed a strong association between the $H L A-B^{*}$ 15:02 allele and the risk of CBZ-induced SJS/TEN in Javanese and Sundanese populations. These findings are similar to those of other studies in Asian populations [2,5,1016. However, there are some reported cases of patients who developed CBZ-induced SJS while screening negative for $H L A-B^{*}$ 15:02. Kaniwa et al. [19] reported not detecting $H L A-B^{*}$ 15:02 in Japanese SJS/TEN patients receiving CBZ, instead finding $H L A-B^{*} 15: 11$, which belongs to the same HLA-B75 serotype. Mehta et al. [16] investigated the association between $H L A-B^{*}$ 15:02 and CBZ-induced SJS in eight Indian patients. While they found that six of the patients did carry $H L A-B^{*} 15: 02$, one patient was homozygous $H L A-B^{*} 15: 08$. Tassaneeyakul et al. (2010) [26] detected $H L A-B^{*} 15: 21$ and $H L A-B^{*} 15: 11$ in a Thai population with CBZ-induced SJS/TEN.

We also identified $H L A-B^{*} 15: 21$ in patients who screened negative for $H L A-B^{*}$ 15:02 but developed SJS/TEN (Table 1). Despite the lack of statistical significance, most likely because of the small patient sample, the finding of $H L A-B^{*} 15: 21$ in our study could indicate possible CBZ-induced SJS/TEN in patients who screen negative for $H L A-B^{*} 15: 02$, especially in the Javanese and Sundanese ethnicities. This suggests that CBZ-induced SJS/TEN may be serotype-specific. However, in this study, we only detected the $H L A-B^{*} 15: 02$ and $H L A-B^{*}$ 15:21 alleles. A recent study from Jaruthamsophon et al. [21] reported a positive association between $H L A-B^{*} 15: 21$ and CBZ-induced SJS/TEN in Asian populations.

Interestingly, in this study, we also found $H L A-B^{*} 18: 01$ and $H L A-B^{*} 44: 03$; both are members of the HLA B44 supertype, the most frequent serotype in our study, and share a peptide-binding specificity [27,28]. Sidney et al. reported that some peptides derived from hepatitis B and C viruses, HIV and Plasmodium falciparum have degenerative B44 supertype-binding capacity [28].

In developing a risk assessment strategy to prevent CBZ-induced SJS/TEN in Indonesia, it would be necessary to take into account the increased likelihood that $\mathrm{B} 75$ serotype alleles contribute to the condition. Further work should be conducted to characterize the role of $H L A-B^{*} 15: 21$ in CBZ-induced SJS/TEN, by expanding this study to other populations. Our findings showed that the HLA-B75 serotype was strongly associated with CBZ-induced SJS/TEN, and was a stronger predictor than $H L A-B^{*}$ 15:02 alone. We suggest that Asians with the HLA-B75 serotype should be considered at-risk for CBZ-induced SJS/TEN, and not only individuals carrying the HLA$B^{*}$ 15:02 allele. Hence, it would be beneficial to develop a diagnostic kit for estimating the risk for CBZ-induced SJS/TEN, especially in Asian populations.

The current study is limited by its small sample size, resulting in a wide CI. Nonetheless, further research is warranted to identify drugs which may induce SJS/TEN, as well as other HLA alleles that can predispose specific populations to SJS/TEN. In conclusion, we have been able to establish an association between the HLA-B75 serotype and CBZ-induced SJS/TEN that is stronger than the well-established association of CBZinduced SJS/TEN with $H L A-B^{*} 15: 02$, in Javanese and Sundanese patients in Indonesia. Our study provides primary evidence that the development of public health recommendations and efficient assays for HLA-75 serotype screening would benefit the cohort under consideration.

\section{Acknowledgements}

The authors would like to thank all YARSI collaborative hospitals for their contributions. They would like to thank the Department of Medical Sciences, Ministry of Public Health Thailand for supporting the HLA-B*15:02 genotyping in this study and T Mushiroda at RIKEN for supplying the reagents for HLA-B genotyping of patients and the control group. They also thank RT Pinzon, S Bahri, IM Noor and R Puspita who supported in sample collection.

Financial \& competing interests disclosure

This study was supported by the US-NIH (grant number UFD5P00010623) and Directorate of Higher Education (DIKTI) grant by Penelitian Produk Terapan scheme (grant number 018/INT/UM/WRII/UY/VII/2017). The authors have no other relevant affiliations or financial involvement with any organization or entity with a financial interest in or financial conflict with the subject matter or materials discussed in the manuscript apart from those disclosed. 
Summary points

- Carbamazepine (CBZ) is common cause of Stevens-Johnson syndrome and toxic epidermal necrolysis (SJS/TEN).

- $H L A-B * 15: 02$ is a well-known genetic marker for CBZ-induced SJS/TEN in Southeast-Asian population.

Materials \& methods

- A total 12 patients of CBZ-induced SJS/TEN, 17 patients of CBZ-tolerant SJS/TEN and unrelated 236 healthy control of Javanese or Sundanese ethnicity were involved in this study.

- HLA-B genotypes were determined using a Luminex multianalyte profiling system (xMAP) with a WAKFlow HLA typing kit (Wakunaga), which is based on polymerase chain reaction-reverse sequence-specific oligonucleotide probes.

Results

- Our result supports the role of $H L A-B * 15: 02$ in CBZ-induced SJS/TEN that was reported in other Asian population.

- We found that $H L A-B * 15: 02$ and $H L A-B * 15: 21$, classified as HLA B-75 serotype, related to CBZ-induced SJS/TEN in Indonesian population.

Conclusion

- We found significant association between B-75 serotype and CBZ-induced SJS/TEN.

- Screening of HLA-B75 serotype is suggested to prevent SJS/TEN in Southeast-Asian population, instead of $H L A-B * 15: 02$ only.

No writing assistance was utilized in the production of this manuscript.

\section{Ethical conduct of research}

The authors state that they have obtained appropriate institutional review board approval or have followed the principles outlined in the Declaration of Helsinki for all human or animal experimental investigators. In addition, for investigations involving human subjects, informed consent has been obtained from the participants involved.

\section{Open access}

This work is licensed under theAttribution-NonCommercial-NoDerivatives 4.0 Unported License. To view a copy of this license, visit http://creativecommons.org/licenses/by-nc-nd/4.0/

\section{References}

1 Ferrell PBJ, McLeod HL. Carbamazepine, $H L A-B^{*} 1502$ and risk of Stevens-Johnson syndrome and toxic epidermal necrolysis: US FDA recommendations. Pharmacogenomics 9(10), 1543-1546 (2009).

2 Zhang Y, Wang J, Zhao L-M, Peng W et al. Strong association between $H L A-B^{*} 1502$ and carbamazepine-induced Stevens-Johnson syndrome and toxic epidermal necrolysis in mainland Han Chinese patients. Eur. J. Clin. Pharmacol. 67(9), 885-887 (2011).

3 Porter NC. Principles and practice of stereotactic radiosurgery. In: Trigeminal Neuralgia: Medical Management Perspective. Chin LS, Regine WF (Eds). Springer Science + Business Media, NY, USA, 535-539 (2008).

4 Hung S, Chung W, Jee $S$ et al. Genetic susceptibility to carbamazepine-induced cutaneous adverse drug reactions. Pharmacogenet. Genomics 16(4), 297-306 (2006).

5 He X, Jian L, He X et al. Association between the $H L A-B^{*}$ 15:02 allele and carbamazepine-induced Stevens-Johnson syndrome/toxic epidermal necrolysis in Han individuals of northeastern China. Pharmacol. Reports 65, 1256-1262 (2013).

6 Kulkantrakorn K, Tassaneeyakul W, Prabmechai N et al. HLA-B*1502 strongly predicts Stevens-Johnson syndrome and toxic epidermal necrolysis in thai patients with neuropathic. Pain Pract. 12(3), 202-208 (2012).

7 Mockenhaupt M. Stevens-Johnson syndrome and toxic epidermal necrolysis: clinical patterns, diagnostic considerations, etiology and therapeutic management. Front. Med. Commun. 33, 10-16 (2014).

8 Chung WH, Hung SI, Hong SH et al. Medical genetics: a marker for Stevens-Johnson syndrome. Nature 428(6982), 486 (2004).

9 Tangamornsuksan W, Chaiyakunapruk N, Somkrua R, Lohitnavy M, Tassaneeyakul W. Relationship between the HLA-B*1502 allele and carbamazepine-induced Stevens-Johnson syndrome and toxic epidermal necrolysis: a systematic review and meta-analysis. JAMA Dermatol. 149, 1025-1032 (2013).

10 Chang C, Too C, Murad S, Hussein SH. Pharmacology and therapeutics association of $H L A-B{ }^{*} 1502$ allele with carbamazepine-induced toxic epidermal necrolysis and Stevens-Johnson syndrome in the multiethnic Malaysian population. Int. J. Dermatol. 50, 221-224 (2011).

11 Dong D, Sung C, Finkelstein A. Cost-effectiveness of $H L A-B^{*} 1502$ genotyping in adult patients with newly diagnosed epilepsy in Singapore. Neurology 79, 1259-1267 (2012). 
12 Man CBL, Kwan P, Baum L et al. Association between $H L A-B^{*} 1502$ allele and antiepileptic drug-induced cutaneous reactions in Han Chinese. Epilepsia 48(5), 1015-1018 (2007).

13 Locharernkul C, Loplumlert J, Limotai C et al. Carbamazepine and phenytoin-induced Stevens-Johnson syndrome is associated with $H L A-B^{*} 1502$ allele in Thai population. Epilepsia 49(12), 2087-2091 (2008).

14 Sun D, Yu C, Liu Z et al. Association of $H L A-B^{*} 1502$ and ${ }^{*} 1511$ allele with antiepileptic drug-induced Stevens-Johnson syndrome in central China. J. Huazhong Univ. Sci. Technol. 34(1), 146-150 (2014).

15 Chen P, Lin J-J, Lu C-S et al. Carbamazepine-induced toxic effects and HLA-B*1502 screening in taiwan. N. Engl. J. Med. 364, 1126-1133 (2011).

16 Mehta TY, Prajapati LM, Mittal B et al. Association of $H L A-B^{*} 1502$ allele and carbamazepine-induced Stevens-Johnson syndrome among Indians. Indian J. Dermatol. Venereol Leprol. 75(6), 579-582 (2009).

17 Lonjou C, Thomas L, Borot N et al. A marker for Stevens-Johnson syndrome. . . : ethnicity matters. Pharmacogenomics J. 6, 265-268 (2006).

18 Kaniwa N, Saito Y, Aihara M et al. HLA-B locus in Japanese patients with antiepileptics and allopurinol-related Stevens-Johnson syndrome and toxic epidermal necrolysis. Pharmacogenomics 9, 1617-1622 (2008).

19 Kaniwa N, Saito Y, Aihara M et al. $H L A-B^{*} 1511$ is a risk factor for carbamazepine-induced Stevens-Johnson syndrome and toxic epidermal necrolysis in Japanese patients. Epilepsia 51(12), 2461-2465 (2010).

20 Gunathilake KMD, Wettasinghe KT, Dissanayake VHW. A study of $H L A-B^{*}$ 15:02 in a Sri Lankan population: implications for pharmacogenomic testing. Hum. Immunol. 77, 429-431 (2016).

21 Jaruthamsophon K, Tipmanee V, Sangiemchoey A, Sukasem C, Limprasert P. HLA-B*15:21 and carbamazepine-induced Stevens-Johnson syndrome: pooled-data and in silico analysis. Sci. Rep. 7, 45553 (2017).

22 Marsh SGE, Albert ED, Bodmer WF et al. Nomenclature for factors of the HLA system, 2010. Tissue Antigens 75(4), 291-455 (2010).

23 Yuliwulandari R, Kashiwase K, Nakajima H et al. Polymorphisms of HLA genes in western Javanese (Indonesia): close affinities to Southeast-Asian populations. Tissue Antigens 73(1), 46-53 (2009).

24 Roujeau JC. The spectrum of SJS/TEN: a clinical classification. J. Invest. Dermatol. 102, 28S-30S (1994).

25 Yuliwulandari R, Sachrowardi Q, Nakajima H et al. Association of HLA-A, -B and -DRB1 with pulmonary tuberculosis in western Javanese Indonesia. Hum. Immunol. 71(7), 697-701 (2010).

26 Tassaneeyakul W, Tiamkao S, Jantararoungtong T et al. Association between $H L A-B^{*} 1502$ and carbamazepine-induced severe cutaneous adverse drug reactions in a Thai population. Epilepsia 51(5), 926-930 (2010).

27 Rist MJ, Theodossis A, Croft NP et al. HLA peptide length preferences control CD8 ${ }^{+}$T-cell responses. J. Immunol. 191, 561-571 (2013).

28 Sidney J, Southwood S, Pasquetto V, Sette A. Simultaneous prediction of binding capacity for multiple molecules of the HLA B44 supertype. J. Immunol. 171, 5964-5974 (2003). 